\title{
An evolutionary analysis of the reaction mechanisms of photosystem I reduction by cytochrome $c_{6}$ and plastocyanin
}

\author{
Miguel A. De la Rosa, José A. Navarro, Antonio Díaz-Quintana, Berta De la Cerda, Fernando P. \\ Molina-Heredia, Alexis Balme, Piedad del S. Murdoch, Irene Díaz-Moreno, Raúl V. Durán and \\ Manuel Hervás \\ Instituto de Bioquímica Vegetal y Fotosíntesis, Universidad de Sevilla y Consejo Superior de \\ Investigaciones Científicas (CSIC), Américo Vespucio s/n, E-41092 Seville, Spain
}

\begin{abstract}
Photosystem I reduction by the soluble metalloproteins cytochrome $c_{6}$ and plastocyanin, which are alternatively synthesized by some photosynthetic organisms depending on the relative availability of copper and iron, has been investigated in cyanobacteria, green algae and plants. The reaction mechanism is classified in three different types on the basis of the affinity of the membrane complex towards its electron donor protein. The role of electrostatic interactions in forming an intermediate transient complex, as well as the structural and functional similarities of cytochrome $c_{6}$ and plastocyanin are analysed from an evolutionary point of view. The proposal made is that the heme protein was first "discovered" by nature, when iron was much more abundant on the Earth's surface, and replaced by plastocyanin when copper became available because of the oxidizing conditions of the new atmosphere.
\end{abstract}

Author Keywords: Cytochrome $c_{6}$; Plastocyanin; Photosystem; Electron transfer

Abbreviations: Cyt, cytochrome $c_{6}$; Fd, ferredoxin; Fld, flavodoxin; $K_{\mathrm{A}}$, equilibrium constant for complex association; $k_{\text {et }}$, electron transfer rate constant; $K_{\mathrm{R}}$, equilibrium constant for complex rearrangement; $\mathrm{PC}$, plastocyanin; PSI, photosystem I; PSII, photosystem II

\section{Introduction}

Photosystem I (PSI) is a membrane-embedded protein complex that carries out the light energy-driven transfer of electrons from a soluble metalloprotein, located inside the thylakoid lumen, to another soluble protein in the outer side of the vesicle (that is, the chloroplast stroma in eukaryotic organisms) [1]. The donor molecule inside the thylakoids can be either the copper protein plastocyanin (Pc) or the heme protein cytochrome $c_{6}(\mathrm{Cyt})$, whereas the acceptor molecule on the other side of the membrane can be either the iron-sulfur protein ferredoxin (Fd) or the flavin-containing protein flavodoxin (Fld) [2].

Some organisms contain the genetic information to make only one of the two alternative proteins ( $\mathrm{Pc}$ or Cyt, Fd or Fld), but some others are able to produce both of them. In the latter organisms, whichever is the protein synthesized depends on the bioavailability of the constituent metal elements. In the particular case of Pc and Cyt, there are some cyanobacteria (probably, the oldest ones) that only contain Cyt, some others are able to make Pc and Cyt, as do green algae as well, and plants just produce Pc [ 3 and 4].

Highly interesting are the evolutionary aspects of the alternative role played by Pc and Cyt. A few years ago, we proposed that Cyt was used by the first oxygen-evolving photosynthetic organisms, when iron was much more available than copper because of the reducing character of the Earth's atmosphere [5]. In fact, the solubility of iron compounds is lower when the metal is in its oxidized state, whereas the solubility of copper substances is higher when the metal is in its oxidized form. As the atmospheric molecular oxygen concentration was rising because of the photosynthetic activity, the relative bioavailabilities of copper and iron were going up and down, respectively, and Cyt was being replaced with Pc. The reason why some cyanobacteria and green algae still keep their capacity to synthesize 
both $\mathrm{Pc}$ and Cyt could be related to their metabolic adaptability to changing environments in seas, lakes and rivers.

If we take into account that PSI evolved from the only photosystem present in anoxygenic green sulfur bacteria, whose electron donor is a c-type cytochrome [6 and 7], it makes sense that such a heme protein adapted itself as to be recognized by the emerging PSI. The other photosystem of oxygenic organisms (PSII)—which possesses a heme protein called cytochrome $c_{549}$, associated to its internal membrane side only in cyanobacteria but not in plants [8 and 9]—should have followed a similar evolutionary process from non-oxygenic purple bacteria, whose electron donor is a mono- or tetra-heme $c$-type cytochrome [6 and 7]. It thus seems that the chlorophyll-containing membrane complex-along with its electron-donating cytochrome-was first evolving from photosynthetic bacteria to cyanobacteria following a divergent pathway up to yield the two photosystems, PSI and PSII. In the course of the further transition from cyanobacteria to green algae and plants, Cyt would be replaced by Pc as electron donor to PSI and cytochrome $c_{549}$ would lose its primitive role up to be totally lacking in eukaryotic PSII, in which it is replaced by a $23-k D a$ protein.

\section{Structural similarities between cytochrome $c_{6}$ and plastocyanin}

Cyt is a $c$-type cytochrome, with a heme group as redox center and a tertiary structure made by three to four alpha-helices [10], whereas Pc is a type I blue copper protein, which consists of several betastrands and a small alpha-helix (see [11], for a review). The geometry of the metal coordination sphere is octaedric for the iron and tetraedric for the copper, and the amino acids acting as metal ligands are histidine and methionine in Cyt [10], and two histidines, one cysteine and one methionine in Pc [11]. An obvious question arises: how can two proteins so much different play the same physiological role? When comparing the physicochemical features of these two proteins, one realizes that they do share a number of crucial parameters: the redox potential value is almost identical (ca. $350 \mathrm{mV}$, at pH 7), the molecular mass is quite similar $(8-10 \mathrm{kDa})$, and the isoelectric point ( $\mathrm{pl}$ ) may vary among the different organisms, but it is practically the same within each organism (Table 1).

Even more interesting is the comparative analysis of the functional areas of the two proteins. Cyt contains a series of hydrophobic residues surrounding the solvent accessible part of the heme group, and $\mathrm{Pc}$ exhibits a similar hydrophobic patch close to the surface histidine coordinating the copper atom. In addition, Pc and Cyt possess an electrostatically charged area-similarly placed at the surface with respect to their respective hydrophobic region-formed by acid or basic residues according to their isoelectric point (Fig. 1). It has been proposed that the electrostatic patch (or site 2) is responsible for complex formation with PSI, and the hydrophobic area is making the close contact with PSI to establish the electron transfer pathway to the oxidized chlorophyll molecule $\mathrm{P} 700^{+}$(see [12], for a recent review). A highly interesting finding showing the close similarity between $\mathrm{Pc}$ and Cyt has recently been achieved with the Anabaena proteins [13]. It has been observed that the only arginyl residue in $\mathrm{Pc}$ and Cyt, which is similarly located at the molecular surface between sites 1 and 2 (Fig. 2), makes the two proteins unable to reduce PSI when it is substituted by glutamate. Furthermore, the replacement of just one aspartate residue — which is similarly located at the edge of site 2 in Pc and Cyt-with a positively charged amino acid enhances the PSI reduction rate with the two proteins [13].

\section{Mechanisms of photosystem I reduction}

The structural similarities between Pc and Cyt can thus explain their ability to interact with PSI, but the differences in their electrostatic area can make them exhibit different affinity towards PSI. This is a key point to interpret the reaction mechanism followed by the two proteins to transfer electrons to the 
oxidized photosystem, according to a process that should involve formation of a transient complex between the two redox partners, rearrangement of the redox centers inside the complex to accommodate themselves in the right position, and electron transfer itself [14]. Assuming that the reaction is triggered by light, the state of PSI when it is excited by the photon will determine the further course of the reaction. Obviously, the different affinity between the donor protein and PSI in the dark will determine that the photosystem is either alone or forming a transient complex-which can be or cannot be properly oriented-when the photoreaction starts.

Fig. 3 displays the three possible routes that would thus lead from the separate reactants to the final products. Which one is the route followed will depend on the relative values of the equilibrium constants for complex formation $\left(K_{\mathrm{A}}\right)$ and complex rearrangement $\left(K_{\mathrm{R}}\right)$ before light excitation, that is, on the predominant state of PSI in the dark. According to this scheme, we have classified the reaction mechanism of PSI reduction by Pc and Cyt in three types, as follows: type I, when $K_{\mathrm{A}}$ is relatively low; type II, when $K_{\mathrm{A}}$ is high but $K_{\mathrm{R}}$ is low; and type III, when both $K_{\mathrm{A}}$ and $K_{\mathrm{R}}$ are high. The rate constant for the overall reaction will thus be determined not only by the relative values of these two equilibrium constants in the dark $\left(K_{\mathrm{A}}\right.$ and $\left.K_{\mathrm{R}}\right)$ and in the light $\left(K_{\mathrm{A}}{ }^{\prime}\right.$ and $\left.K_{\mathrm{R}}{ }^{\prime}\right)$ but also by the rate of electron transfer $\left(k_{\mathrm{et}}\right)$ after PSI photooxidation. Even though such a theoretical model comprises all possible equilibria, the kinetic analyses of experimental data can easily be performed according to a much simpler model, as described by Hervás et al. [14].

\section{Electrostatic and hydrophobic interactions}

The affinity of Pc and Cyt towards PSI is a key parameter in the description of the whole process, as likewise is the flexibility of the two partners inside the transient complex. In this context, it is easy to understand that the surface electrostatic potential distribution and the hydrophobic areas are as important as the steric factors to allow for the right accommodation of the reactants like two pieces of a puzzle. In fact, the nature and intensity of long-range interactions between PSI and its donor protein will govern the formation of the transient complex, whereas the short-range forces along with the steric adjustments will mainly control the complex rearrangement and electron transfer.

In the last years, we have investigated the reaction mechanism of PSI photoreduction in a number of evolutionarily distant organisms, namely cyanobacteria, green algae and plants $[13,14,15,16,17$ and 18]. Table 1 summarizes the isoelectric point of Pc and Cyt in every organism. The electrostatic charge of these two proteins_-and that of PSI as well-actually makes the reactants interactions repulsive (Synechocystis and Phormidium) [16 and 17], neutral (Prochlorothrix Pc) [18] or attractive (others) [13, 14 and 15]. In the particular case of Synechocystis and Phormidium, the repulsive nature of the interactions between PSI and its donor protein explains why PSI reduction follows a type I mechanism. The Prochlorothrix Pc, in its turn, reacts according to a type II mechanism, with formation of an intermediate complex that is stabilized by hydrophobic forces. In other organisms, it is possible to observe the existence of an electrostatic intermediate complex, independently of the fact that the further rearrangement could be (type III) or not be (type II) kinetically detected.

The long evolution of oxygenic photosynthetic organisms - the first cyanobacteria appeared on the Earth's surface more than three billion years ago-would thus lead the reaction mechanism from the type I to type III, through type II, in order to reach the maximum efficiency of PSI reduction. However, such a unidirectional process should have followed some discontinuous ways because of the transition from Cyt to Pc. It is indeed possible that the mechanism of PSI reduction was first being adapted so as to evolve from type I to type III with Cyt, but the further appearance of Pc required a second adaptation of PSI to its new electron donor protein. This is thus an excellent case study of an evolutionary process 
that is both convergent (two different proteins adapting each other to play the same physiological role within each organism) and parallel (two different proteins evolving similarly from one to another organism) [2].

\section{Breaking-off of the intermediate complex}

The redox reaction should not be considered to be complete after electron transfer but after dissociation of the oxidized donor protein from its binding site in PSI, thereby leaving it free for another reduced molecule to bind. In the case of type I mechanism, the repulsive interactions between PSI and its donor protein impede the formation of any stable transient complex-the reaction rather proceeds according to an oriented collision between the soluble donor protein and the membrane embedded PSI; it is not so difficult to envisage that a new reaction could easily start when a second molecule approximates to PSI. Even in the case of the type II mechanism involving hydrophobic recognition between the partners, the rather low intensity of such interactions could readily destabilize the complex.

In the case of type II and III mechanisms with electrostatically stabilized transient complexes, the dissociation of partners can be favoured by the shift of a negative charge from the metal atom to the chlorophyll molecule and the subsequent electron density displacement in the interaction area, thereby inducing small but crucial conformational changes that could trigger complex breaking-off. In this context, it is worth noting that the solution structure of Monoraphidium Cyt reveals interesting differences between its oxidized and reduced states (Fig. 4). In fact, one of the two propionates of the heme group, as well as the side chains of some amino acids - in particular, histidine at position 30-are significantly displaced depending on the redox state of the heme iron [19]. Similar structural changes between the reduced and oxidized states of $\mathrm{Pc}_{\mathrm{c}}$ have been likewise observed [20 and 21].

\section{Concluding remarks}

In summary, we can say that PSI reduction by Pc and Cyt has experienced a long evolutionary process in order to not only optimise the kinetic efficiency of the overall reaction, but also to promote the transition from Cyt to $\mathrm{Pc}$. At the beginning, the molecular recognition and complex formation were not well established, but the subsequent evolution made the attractive electrostatic interactions the factors governing the redox reaction up to optimise the electron transfer within a rather stable transient complex. Finally, local structural changes in the interaction area between the two partners were designed to reach the last stage - that is, the complex dissociation after electron transfer-and start a new redox cycle.

\section{Acknowledgements}

The research was supported by the European Union (Networks ERB-FMRX-CT98-0218 and HPRNCT1999-00095), Spanish Ministry of Science and Technology (MCYT, Grant BMC2000-0444), and Andalusian Government (PAI, CVI-0198).

\section{References}

1. P.R. Chitnis, Photosystem I. Plant Physiol. 111 (1996), pp. 661-669.

2. J.A. Navarro, M. Hervás and M.A. De la Rosa, Co-evolution of cytochrome $c_{6}$ and plastocyanin, mobile proteins transferring electrons from cytochrome $b_{6} f$ to photosystem I. J. Biol. Inorg. Chem. 2 (1997), pp. 11-22

3. G. Sandmann, Formation of plastocyanin and cytochrome $c_{553}$ in different species of blue-green algae. Arch. Microbiol. 145 (1986), pp. 76-79.

4. G. Sandmann, H. Reck, E. Kessler and P. Böger, Distribution of plastocyanin and soluble plastidic cytochrome $c$ in various classes of algae. Arch. Microbiol. 134 (1983), pp. 23-27. 
5. R.J.P. Williams and J.J.R. Fraústo da Silva The Natural Selection of the Chemical Elements-The Environment and Life's Chemistry, Clarendon Press, Oxford (1997).

6. R.E. Blankenship, Origin and early evolution of photosynthesis. Photosynth. Res. 33 (1992), pp. 91111.

7. J.P. Allen and J.C. Williams, Photosynthetic reaction centers. FEBS Lett. 438 (1998), pp. 5-9.

8. C. Frazao, F.J. Enguita, R. Coehlo, G.M. Sheldrick, J.A. Navarro, M. Hervás, M.A. De la Rosa and M.A. Carrondo, Crystal structure of low-potential cytochrome $c_{549}$ from Synechocystis sp. PCC 6803 at $1.21 \AA ̊$ resolution. J. Biol. Inorg. Chem. 6 (2001), pp. 324-332.

9. Zouni, H.T. Witt, J. Kern, P. Fromme, N. Krauß, W. Saenger and O. Orth, Crystal structure of photosystem II from Synechococcus elongatus at 3,8 Å resolution. Nature 409 (2001), pp. 739-743.

10. Frazao, C.M. Soares, M.A. Carrondo, E. Pohl, Z. Dauter, K.S. Wilson, M. Hervás, J.A. Navarro, M.A. De la Rosa and G. Sheldrick , Ab initio determination of the crystal structure of cytochrome $c_{6}$; comparison with plastocyanin. Structure 15 (1995), pp. 1159-1169.

11. K. Sigfridsson, Plastocyanin: an electron-transfer protein. Photosynth. Res. 57 (1998), pp. 1-28.

12. A.B. Hope, Electron transfer amongst cytochrome $f$, plastocyanin and photosystem I: kinetics and mechanisms. Biochim. Biophys. Acta 1456 (2000), pp. 5-26.

13. F.P. Molina-Heredia, M. Hervás, J.A. Navarro and M.A. De la Rosa, A single arginyl residue in plastocyanin and in cytochrome $c_{6}$ from the cyanobacterium Anabaena sp. PCC 7119 is required for efficient reduction of photosystem I. J. Biol. Chem. 276 (2001), pp. 601-605.

14. M. Hervás, J.A. Navarro, A. Díaz, H. Bottin and M.A. De la Rosa, Laser-flash kinetic analysis of the fast electron transfer from plastocyanin and cytochrome $c_{6}$ to photosystem I; experimental evidence on the evolution of the reaction mechanism. Biochemistry 34 (1995), pp. 11321-11326.

15. F.P. Molina-Heredia, A. Díaz, M. Hervás, J.A. Navarro and M.A. De la Rosa, Site-directed mutagenesis of cytochrome $c_{6}$ from Anabaena sp. PCC 7119. J. Biol. Chem. 274 (1999), pp. 3356533570.

16. B. De la Cerda, J.A. Navarro, M. Hervás and M.A. De la Rosa, Changes in the reaction mechanism of electron transfer from plastocyanin to photosystem I in the cyanobacterium Synechocystis sp. PCC 6803 as induced by site-directed mutagenesis of the copper protein. Biochemistry 36 (1997), pp. 10125-10130.

17. B. De la Cerda, A. Díaz-Quintana, J.A. Navarro, M. Hervás and M.A. De la Rosa, Site-directed mutagenesis of cytochrome $c_{6}$ from Synechocystis sp. 6803; the heme protein possesses a negatively charged area that may be isofunctional with the acidic patch of plastocyanin. J. Biol. Chem. 274 (1999), pp. 13292-13297.

18. J.A. Navarro, M. Hervás, C.R. Babu, F.P. Molina-Heredia, G.S. Bullerjahn and M.A. De la Rosa In: G. Garab, Editor, Photosynthesis: Mechanisms and Effects, Kluwer Academic Publishing, Dordrecht, The Netherlands (1998), pp. 1605-1608.

19. L. Banci, I. Bertini, M.A. De la Rosa, D. Koulougliotis, J.A. Navarro and O. Walter, The solution structure of oxidized cytochrome $c_{6}$ from the green alga Monoraphidium braunii. Biochemistry 37 (1998), pp. 4831-4843.

20. F. Drepper, M. Hippler, W. Nitschke and W. Haehnel, Binding dynamics and electron transfer between plastocyanin and photosystem I. Biochemistry 35 (1996), pp. 1282-1295.

21. T. Inoue, H. Sugawara, S. Hamanaka, H. Tsukui, E. Suzuki, T. Kohzuma and Y. Kai , Crystal structure determinations of oxidized and reduced plastocyanin from the cyanobacterium Synechococcus sp. PCC 7942. Biochemistry 38 (1999), pp. 6063-6069. 


\section{Table}

Table 1. Variations in the isoelectric point (pl) and in the nature of the interactions with photosystem I of cytochrome $c_{6}$ (left) and plastocyanin (right) isolated from differently evolved organisms

\begin{tabular}{|c|c|c|}
\hline Organism & Isoelectric point (pl) & Interaction ${ }^{3}$ with PSI \\
\hline \multicolumn{3}{|l|}{ Cyanobacteria } \\
\hline Prochlorothrix hollandica & $\sim 8 / 8.4$ & $+/ 0$ \\
\hline $\begin{array}{l}\text { Synechocystis sp. PCC } \\
6803\end{array}$ & $5,6 / 5,5$ & $-1-$ \\
\hline Phormidium laminosum & $5.1 / 5.0$ & $-1-$ \\
\hline Anabaena sp. PCC 7119 & $9.0 / 8.8$ & $+1+$ \\
\hline $\begin{array}{l}\text { Pseudanabaena sp. PCC } \\
6903\end{array}$ & $8,0 / 8.3$ & $+1+$ \\
\hline \multicolumn{3}{|l|}{ Green alga } \\
\hline Monoraphidium braunĭ & $3.6 / 3.7$ & $+1+$ \\
\hline \multicolumn{3}{|l|}{ Plant } \\
\hline Spinacea oleracea & 4,2 & $+/+$ \\
\hline
\end{tabular}

Original data in $[13,14,15,16,17$ and 18]

\section{Figures}

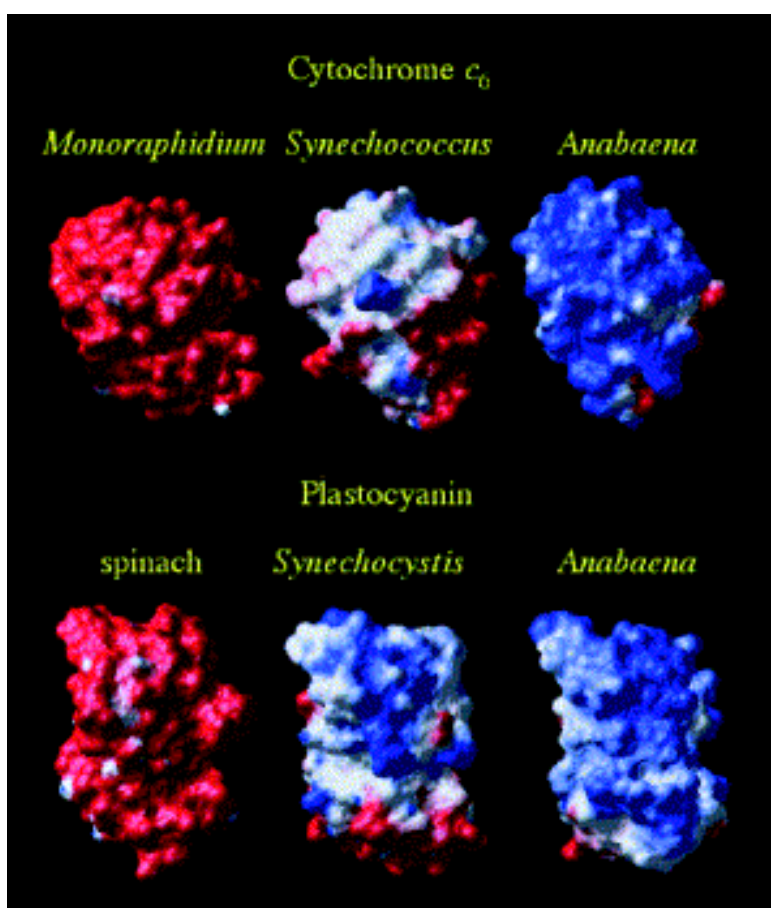

Fig. 1. Surface electrostatic potential distribution of cytochrome $c_{6}$ and plastocyanin from different organisms. The two molecules are oriented with their sites 1 and 2 at the top and in front, respectively. Positively and negatively charged areas are in blue and red, respectively. Calculations were made at 40 $\mathrm{mM}$ ionic strength and $\mathrm{pH} 7$. 


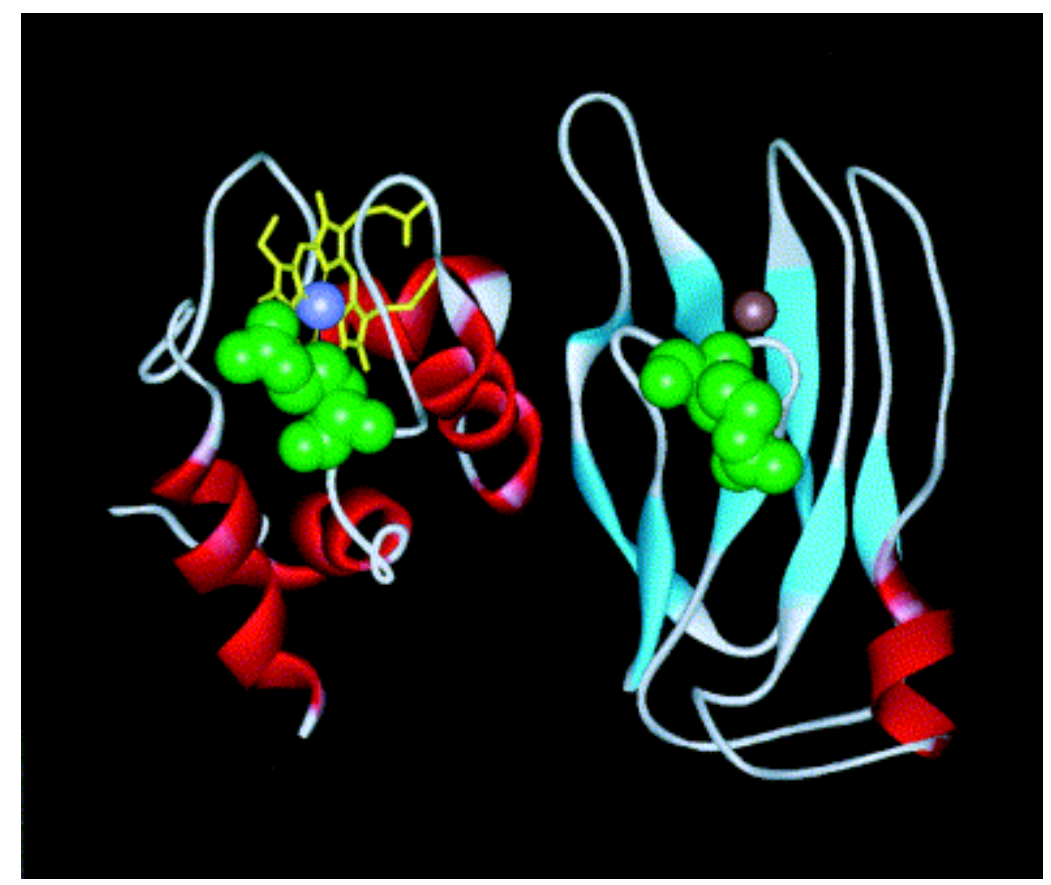

Fig. 2. The only arginyl residue in cytochrome $c_{6}$ (left) and plastocyanin (right) from Anabaena is similarly located between their respective sites 1 and 2. The orientation of the two molecules is the same as in Fig. 1. The arginyl residue is in green.

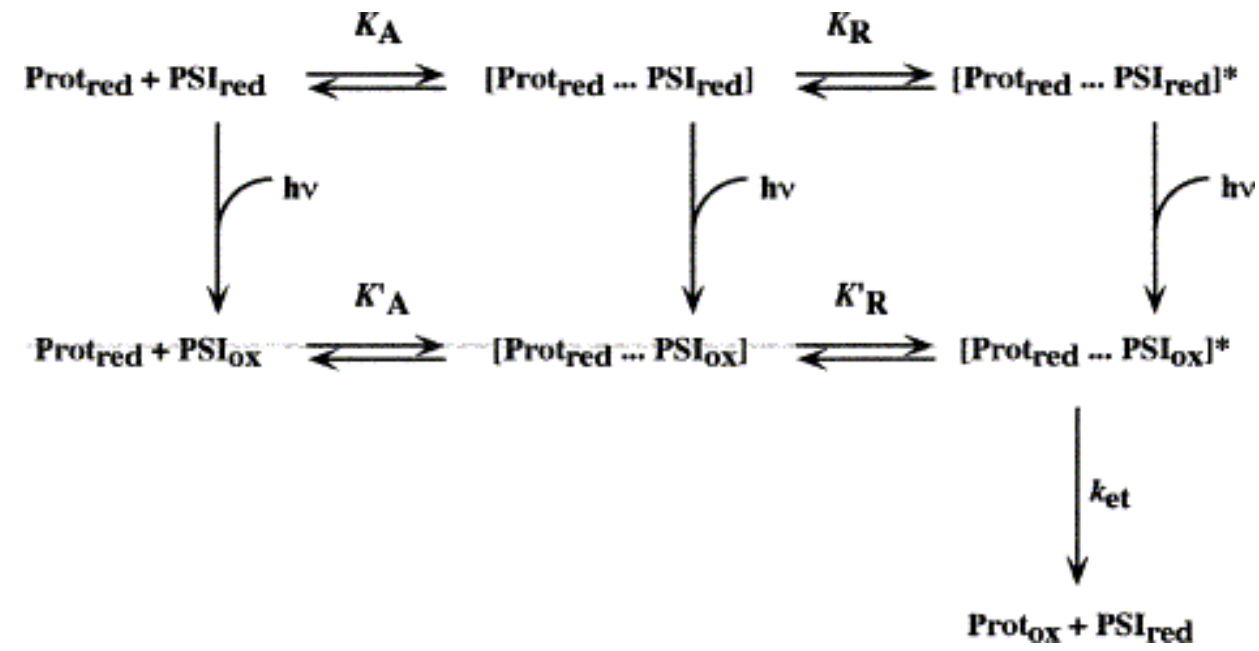

Fig. 3. Reaction mechanisms of photosystem I (PSI) reduction by its electron donor protein (Prot), namely cytochrome $c_{6}$ or plastocyanin. The reaction mechanism is classified in three types depending on the route followed by the reactants. See the text for explanation. 

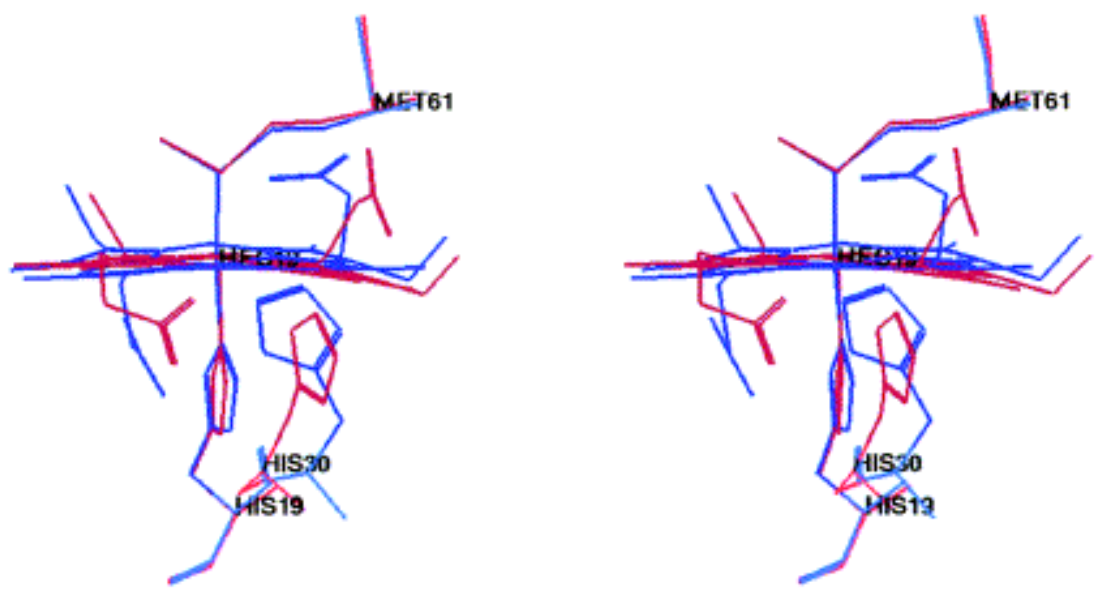

Fig. 4. Stereoview showing the main conformational changes between the oxidized (red) and reduced (blue) forms of Monoraphidium cytochrome $c_{6}$. 